ISSN: 2600-5859

Vol. 4, N¹.1, p. 83-100, Febrero, 2021

\title{
Modelo de gestión sostenible para el manejo de la cuenca hidrográfica "Tenguel Alto"
}

\author{
Sustainable management model for the management of the "Tenguel Alto" \\ water basin
}

Ing. Sebastián Israel Chavez Barriga. ${ }^{1} \&$ Yonimiler Castillo Ortega. ${ }^{2}$

Recibido: 16-12-2020 / Revisado: 06-01-2021 /Aceptado: 14-01-2019/ Publicado: 05-02-2021

\begin{abstract}
.
DOI: https://doi.org/10.33262/concienciadigital.v4i1.1.1548

Introduction: This proposal proposes the development of a sustainable management model for the management of the "Tenguel Alto" watershed, given the need to solve a problem that arises in daily living in the watershed, the information of which plans to generate an instrument for the proper management of the basin. Objectives: Answer the following questions: What are the theoretical trends on the management of sustainable local development with emphasis on the management of hydrographic basins? What are the effects of watersheds on local development? What are the components of the sustainable management model of the Tenguel Alto watershed? What is the validity of the sustainable management model of the river basin according to the criteria of experts? Methodology: To answer these questions, three stages are established: a theoretical analysis, in order to conceptualize and justify the research, followed by the application of a survey to a sample of 378 participants, to know the socioeconomic reality, finally, papers are analyzed and articles published in scientific journals and conferences on the matter in order to establish the management model. Results: It is determined that $70 \%$ have a monthly income less than or equal to the unified basic salary, despite having a hydrographic basin, most of the rural population lacks potable water. Conclusion: Finally, it is concluded, responding to the questions raised, that the EARP management model should focus on an adequate administration of the water formed in the basin through strategies that involve the population in the conservation of water resources, the administration of resources water strengthening the ecosystem.
\end{abstract}

Keywords: Water management, urban management, socio-environmental management. Sustainable management.

\section{Resumen.}

${ }^{1}$ Universidad Católica de Cuenca, Posgrado, Maestría en Desarrollo Local Mención en Ordenamiento Territorial, Cuenca, Ecuador, sebastian.chavez@est.ucacue.edu.ec, https:// orcid.org/0000-0002-9732-2254

${ }^{2}$ Universidad Católica de Cuenca, Posgrado, Maestría en Desarrollo Local Mención en Ordenamiento Territorial, Cuenca, Ecuador, ycastilloo@ucacue.edu.ec, Afiliación: https://orcid.org/0000-0002-7710-5199 
Introducción: Esta propuesta plantea el desarrollo de un modelo de gestión sostenible para el manejo de la cuenca hidrográfica "Tenguel Alto", ante la necesidad de resolver un problema que se suscita en el diario vivir en la cuenca hidrográfica cuya información planea generar un instrumento para el manejo adecuado de la cuenca. Objetivos: Responder las siguientes preguntas: ¿Cuáles son las tendencias teóricas sobre la gestión del desarrollo local sostenible con énfasis en el manejo de cuencas hidrográficas? ¿Cuál son los efectos de las cuencas en el desarrollo local? ¿Cuáles son los componentes del modelo de gestión sostenible de la cuenca hidrográfica del Tenguel alto? ¿Cuál es la validez del modelo de gestión sostenible de la cuenca hidrográfica según el criterio de expertos? Metodología: Para responder dichas preguntas se establecen tres etapas: un análisis teórico, con la finalidad de conceptualizar y justificar la investigación, seguido de la aplicación de una encuesta a una muestra de 378 participantes, para conocer la realidad socioeconómica, finalmente, se analizan ponencias y artículos publicados en revistas científicas y conferencias al respecto para así establecer el modelo de gestión. Resultados: Se determina que el $70 \%$ poseen un ingreso mensual menor o igual al salario básico unificado, pese a tener una cuenca hidrográfica, la mayor parte de la población rural carece de agua potable. Conclusión: Finalmente se concluye, respondiendo a las preguntas planteadas, que el modelo de gestión EARP debe centrarse en una adecuada administración del agua formada en la cuenca mediante estrategias que involucren a la población en la conservación de los recursos hídricos, la administración de los recursos hídricos fortaleciendo el ecosistema.

Palabras claves: Gestión del agua, Gestión urbana, Gestión socioambiental. Gestión Sostenible.

\section{Introducción.}

Martínez Valdés, Y., \& Villalejo García, V. M. (2018) afirman que el agua, como recurso vital para la vida y el desarrollo de cualquier país, debe ser administrada en beneficio de toda la población, lo cual implica asumir responsabilidades relacionadas con su contabilización, conservación y control de uso adecuado, así como reglamentar la asignación de derechos de uso del agua. La gestión del agua tiene que ver con la forma como se administra este recurso natural. Hay que tener en cuenta que si hay o habrá una crisis del agua también habrá una crisis del desarrollo.

La cuenca es reconocida como la unidad territorial más adecuada para la gestión integrada de los recursos hídricos. Según Jouravlev (2009), la validez de lo anteriormente señalado ha sido enfatizada y recomendada en las más importantes conferencias internacionales sobre el agua, tales como: Conferencia de las Naciones Unidad sobre el Agua, Mar del Plata, Argentina; Marzo 1977; Conferencia Internacional sobre el Agua y el Medio Ambiente, CIAMA Dublín, Irlanda; Enero de 1992; Conferencia de las Naciones Unidas sobre el Medio Ambiente y el Desarrollo CNUMAD, Río de Janeiro, Brasil; Junio 1992 y en el Plan de 
Aplicación de la Decisiones de la Cumbre Mundial sobre Desarrollo Sostenible, Johannesburgo, Sudáfrica, Septiembre 2002 .

Considerando que la cuenca es la unidad más apropiada para la gestión integrada de los recursos hídricos (GIRH), así como reconociendo el principio de subsidiariedad como uno de los principios fundamentales de la GIRH (Núñez, 2011), desde hace algún tiempo países de la región como México y Brasil han diseñado la institucionalidad para la gestión del agua basándose en las cuencas hidrográficas como unidades de gestión, aspecto que también se ha incorporado en Ecuador (creación de las demarcaciones hidrográficas en función de la cuencas, Acuerdo Ministerial de SENAGUA Nro. 2010-66) y Perú (Ley 29338, Ley de Recursos Hídricos, Marzo 2009). Aun cuando en los dos últimos casos antes descritos aún subsista la discusión sobre la conformación, atribuciones o competencias de los organismos o entidades de cuenca, algo sobre lo que existe pleno consenso es el de reconocer a la cuenca hidrográfica como la unidad para la gestión del agua. (SENAGUA, 2010) (Congreso Perú, 2009)

La institucionalidad para gestión del agua, basada en cuencas hidrográficas, trasciende inclusive fronteras nacionales (Sadoff, C., Greiber, T., Smith, M. \& Bergkamp, G., 2008), en por lo cual se han creado entidades de carácter binacional para la gestión de recursos hídricos como por ejemplo en Ecuador y Perú con la Comisión binacional para la GIRH en la cuenca del río Zarumilla, en Bolivia y Perú en la cuenca del Titicaca se crea la Autoridad Binacional del sistema TDPS ALT; también encontramos tratados que involucran varias naciones para administrar las de cuencas hidrográficas como ejemplo encontramos el organismo del Tratado de Cooperación Amazónica (OTCA) en el cual están involucrados 8 países.

La GIRH nace como una respuesta a la crisis que se vive en el mundo respecto al manejo de este recurso, esta preocupación no se ha presentado recientemente, sino que ha sido una constante a lo largo de los años a nivel nacional e internacional. La GIRH se inicia como un concepto empírico con base en la experiencia dada por el ejercicio profesional. Sin embargo, solamente después de la Agenda 21 y de la Cumbre Mundial sobre el Desarrollo Sostenible en 1992, es que el concepto de GIRH fue debatido a profundidad. Finalmente, tiene una mayor aceptación a nivel mundial el concepto de GIRH dado por la Asociación Mundial para el Agua: "La GIRH es un proceso a favor del desarrollo y la administración coordinados, principalmente el suelo, del agua y los otros recursos relacionados, con el fin de aumentar los efectos económicos y el bienestar social de forma equitativa sin comprometer la sostenibilidad de los ecosistemas vitales" (PNUMA-DHI, 2017)

Así también se toma en consideración que La sensibilidad desarrollada y amplificada en las últimas décadas sobre el medio ambiente introdujo un nuevo elemento a considerar para la participación comunal/regional. Efectivamente, nuevas cuestiones son parte ahora de la agenda de lucha y reivindicación política de las comunidades locales (o al menos de ciertos grupos comunitarios), destacando los temas ligados a los recursos naturales y el ambiente. 
(Barrientos, 2006) Y en concordancia con lo anterior Peña, Pino, \& De León, (2015).afirman que la gestión ambiental de la cuenca del Río En los últimos tiempos el incremento desmedido de los problemas ambientales y la falta de cultura preservadora, ha dado lugar a reflexionar y tratar de buscar soluciones para atenuar las afectaciones del medioambiente desde sus diferentes enfoques, particularmente en el deterioro desmedido de las cuencas hidrográficas, y por la falta de esfuerzos a gran escala y a la desarticulación de los estamentos involucrados y beneficiados directa e indirectamente del mismo.

La ONU (2017) describe el recurso hídrico como un aspecto determinante para el desarrollo económico y social de la población, a la vez que establece que este recurso debería ser estudiado en el contexto del ecosistema en el que se encuentra pues se considera también esencial para el desarrollo y mantenimiento de otros recursos. Además, considera que el sector privado y gubernamental tienen un papel protagónico en la gestión del recurso hídrico, cuando se encuentran a la cabeza del sistema de GIRH, pues son encargados de tomar decisiones en cuanto a la dotación del recurso, cobro y establecimiento de tasas por el uso y aprovechamiento del agua, así como también enfrentan una realidad problemática en cuanto al uso del recurso, pues la tendencia en el crecimiento demográfica hace que se incremente la demanda del recurso con el paso del tiempo mientras que la producción del mismo disminuye si no se realiza un aprovechamiento adecuado de las fuentes. (Amendaño Estévez, E. N., 2018)

En consecuencia, la ONU recomienda realizar un enfoque holístico para la gestión integrada del recurso hídrico en post de una gestión y desarrollo equitativo, eficiente y sostenible, partiendo de que el recurso agua cada vez es más limitado y la demanda crece con el tiempo. De manera que la gestión integral del recurso hídrico puede considerarse como un proceso sistemático que se representa en el siguiente diagrama (ONU, 2017)

Por lo anteriormente citado, surgen las siguientes preguntas de investigación: ¿Cuáles son las tendencias teóricas sobre la gestión del desarrollo local sostenible con énfasis en el manejo de cuencas hidrográficas? ¿Cuál son los efectos de las cuencas en el desarrollo local? ¿Cuáles son los componentes del modelo de gestión sostenible de la cuenca hidrográfica del Tenguel alto? ¿Cuál es la validez del modelo de gestión sostenible de la cuenca hidrográfica según el criterio de expertos?

La investigación plantea el desarrollo de un modelo de gestión sostenible para el manejo de la cuenca hidrográfica "Tenguel Alto", ante la necesidad de resolver un problema que se suscita en el diario vivir en la cuenca hidrográfica cuya información planea generar un instrumento para el manejo adecuado de la cuenca.

En la cuenca hidrográfica "Tenguel Alto" se integran sistemas económicos, biofísicos, políticos y administrativos. Dentro de un desarrollo socioeconómico, los distintos tipos de cuencas hidrográficas se componen de un sistema humano y biofísico, en el cual los causes 
ISSN: 2600-5859

www.concienciadigital.org

Vol. 4, N¹.1, p. 83-100, Febrero, 2021

de agua determinan un papel importante en el entorno, ya que estos pueden ser modificados por los hombres para sus intereses personales o a la vez sociales, pero las leyes físicas no van a cambiar por lo que se debe respetar el ecosistema de una cuenca hidrológica. El manejo de socio ecosistemas debe descansar en acuerdos sociales que respeten los procesos biológicos y se dobleguen ante las leyes fisicoquímicas (Maass, 2015, p 7)

La cuenca hidrográfica "Tenguel Alto" (Figura 1) se ubica en el cantón Camilo Ponce Enríquez, en la provincia del Azuay, el cual está sujeto a distintas presiones por la demanda de agua. Para poder satisfacer todas y cada una de estas necesidades dependen de la cuenca hidrográfica más cercana a la cabecera cantonal y que esta cumpla con los parámetros requeridos para ser potabilizados, riego, o uso minero. La cuenca hidrográfica que cumple con todas las características necesarias es la de "Tenguel Alto". La problemática principal es el mal manejo de la cuenca de la cual se abastece de líquido vital a la población del centro cantonal y de sus comunidades aledañas. Esto se debe a la presencia minera, al exceso de bocaminas ilegales y sin estudios técnicos por lo que está provocando la disminución de caudal superficial, entre las principales comunidades que se desarrollan en esta cuenca esta: La rica, Villa Rica, San Francisco de Muyuyacu, San Vicente de Cachi, Moras, Guena, San Gerardo, La Unión, San Juan de Naranjillas, El Progreso

Figura 1. Cuenca hidrográfica "Tenguel Alto

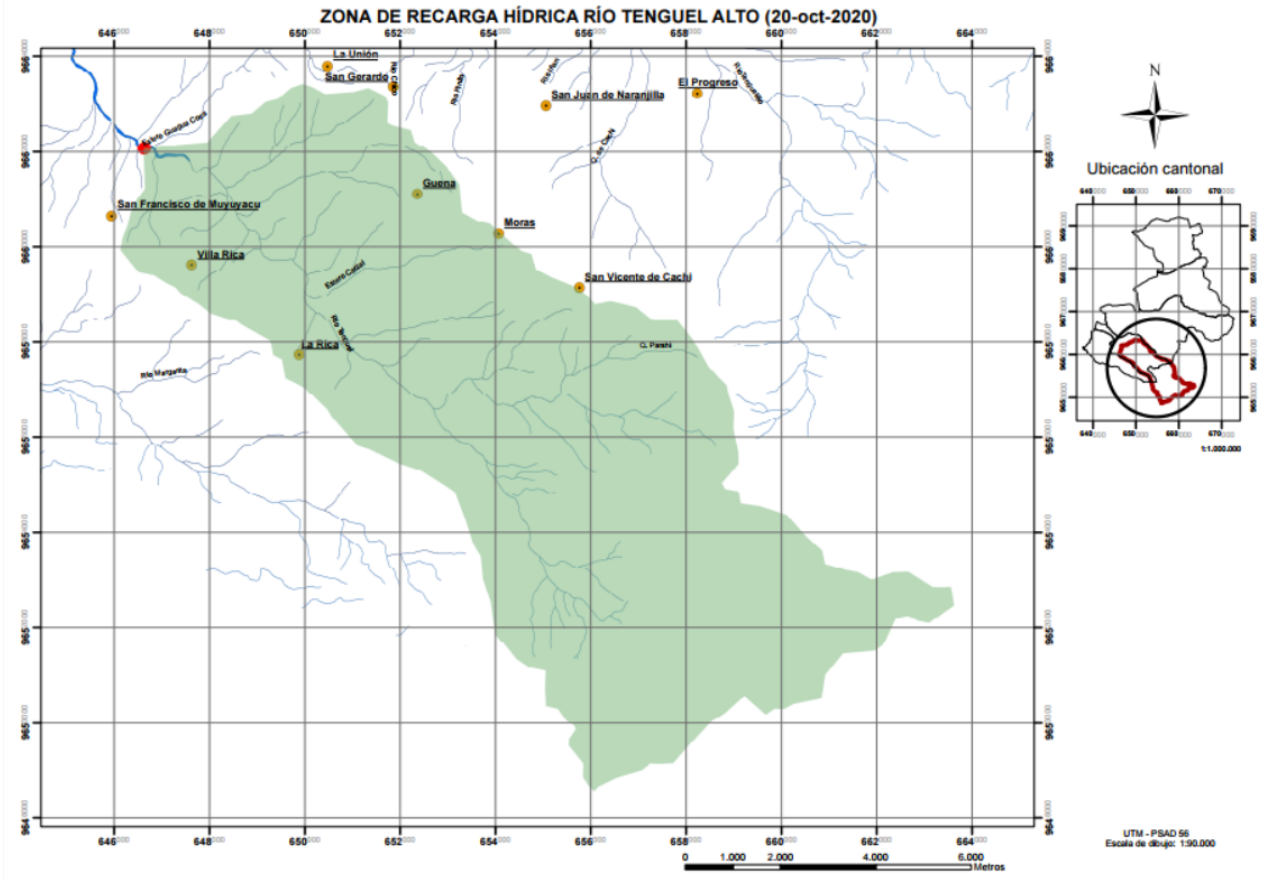

Fuente: El autor

\section{Metodología.}

Para responder las preguntas de investigación planteadas, se planifica una metodología de 3 etapas: 
ISSN: 2600-5859

Vol. 4, N¹.1, p. 83-100, Febrero, 2021

Primera etapa. Recopilación de información: Esta etapa se la ejecutó para conceptualizar y dar a conocer la importancia de realizar la presente investigación, caracterizar la cuenca hidrográfica, relieve, y comunidades aledañas.

Segunda etapa. Encuesta. Se aplica una encuesta para conocer la realidad socioeconómica de la población cercana a la Cuenca hidrográfica Tenguel alto, la cual se encuentra en el Cantón Camilo Ponce Enríquez, cuenta con una población es 21998 habitantes, al aplicar los siguientes la formula estadística se encuentra una muestra de 378 personas, se aplicaron los siguientes ítems: nivel de heterogeneidad del $50 \%$ un margen de error de $0,05 \%$ y un nivel de confianza de $95 \%$

En la tercera etapa mediante el estudio de publicaciones en revistas y conferencias se llegan a establecer los puntos que debe examinar el modelo de gestión sostenible para el manejo de la cuenca hidrográfica "Tenguel Alto", en dicho análisis se estudia la bibliografía disponible sobre modelos de gestión y sobre la gestión de cuencas hidrográficas. Dando de esta manera los factores idóneos a tomar en cuenta para una gestión sostenible aplicable a la cuenca hidrográfica "Tenguel Alto". Se propone un modelo que es validado por expertos, basado en el método Delphi.

Tabla 1. Metodología

\begin{tabular}{|c|c|}
\hline ASPECTO & EXPLICACIÓN \\
\hline $\begin{array}{l}\text { Preguntas de } \\
\text { investigación }\end{array}$ & $\begin{array}{l}\text { Esta investigación busca responder a las siguientes preguntas de inves } \\
\text { ¿Cuáles son las tendencias teóricas sobre la gestión del desarrollo loca } \\
\text { con énfasis en el manejo de cuencas hidrográficas? } \\
\text { ¿Cuál son los efectos de las cuencas en el desarrollo local? } \\
\text { ¿Cuáles son los componentes del modelo de gestión sostenible de la cı } \\
\text { hidrográfica del Tenguel alto? } \\
\text { ¿Cuál es la validez del modelo de gestión sostenible de la cuenca hidrc } \\
\text { según el criterio de expertos? }\end{array}$ \\
\hline $\begin{array}{l}\text { Estrategias de } \\
\text { investigación }\end{array}$ & $\begin{array}{l}\text { Área: Desarrollo local, gestión sostenible, manejo de cuencas hidrográ } \\
\text { Propósito de la búsqueda: Determinar los factores más relevantes para } \\
\text { de gestión sostenible de la cuenca hidrográfica del Tenguel alto }\end{array}$ \\
\hline $\begin{array}{l}\text { Fuentes de } \\
\text { información }\end{array}$ & Tesis, Artículos científicos, Libros \\
\hline $\begin{array}{l}\text { Motores de } \\
\text { búsqueda }\end{array}$ & Google Scholars, Scielo, Scopus, \\
\hline $\begin{array}{l}\text { Criterios de } \\
\text { búsqueda }\end{array}$ & Desarrollo local, gestión sostenible, manejo de cuencas hidrográficas. \\
\hline $\begin{array}{l}\text { Criterios de } \\
\text { Inclusión }\end{array}$ & $\begin{array}{l}\text { Documentos que contienen información sobre nuestras preguntas de } \\
\text { investigación }\end{array}$ \\
\hline $\begin{array}{l}\text { Criterios de } \\
\text { Exclusión }\end{array}$ & Se excluyen los documentos no referentes al ítem anterior \\
\hline
\end{tabular}


Evaluación del contenido de los criterios información
Exactitud, objetividad, cobertura, relevancia de acuerdo con las preguntas de investigación.

Se investiga en la cadena de autores que han realizado contribuciones a los ítems vistos.

Se define una visión general de análisis de la importancia de las cuencas hidrográficas y la realización de un plan de gestión integral con el fin de establecer los factores clave que pueden ser relevantes para el modelo de gestión sostenible de la cuenca hidrográfica del Tenguel alto

Elaborado por: El Autor

\section{Resultados.}

El modelo, desarrollado para la investigación se denomina EARP (Enfoque, Análisis, Requerimiento, Planificación) está establecido por 4 objetivos, en los cuales se darán tareas en las que participan los actores clave que involucra a los mineros de la zona, a la ciudadanía en general y al GAD del cantón camilo Ponce Enríquez; también se propone que académicos de las universidades de la zona puedan colaborar en la gestión integral mediante la investigación en cada uno de los puntos que componen el modelo EARP, y que se detallan a continuación:

- Enfoque de la gestión integral sostenible de las cuencas hidrográficas

- Análisis socioeconómico:

- Requerimientos de una política integral

- Planificación de gestión integral sostenible

\section{a. Enfoque de la gestión integral sostenible de las cuencas hidrográficas}

La adecuada gestión del manejo de cuencas hidrográficas debe conducir al desarrollo sustentable lo cual se e refiere más bien a la posibilidad de mantener un equilibrio entre factores que implican un cierto nivel de desarrollo del ser humano, nivel que es siempre transitorio, en evolución y, al menos en teoría, debería ser siempre conducente a mejorar la calidad de vida de los seres humanos.

El concepto de desarrollo sustentable y sostenible está además vinculado a categorías o escalas de calidad de vida y a la interacción entre habitantes de uno o más territorios que intercambian recursos o migran de un lugar a otro.

El manejo integral de cuencas hidrográficas se define como "la gestión que el hombre realiza en un determinado sistema hidrográfico para aprovechar y proteger los recursos naturales que le ofrece con el fin de obtener una producción optima y sostenida" (Gaspari et al., 2009) Este tipo de gestión otorga al desarrollo sustentable un ámbito geográfico de aplicación que incluye un proceso que consiste en planear, organizar, dirigir, evaluar y controlar la ejecución 
de sus acciones preservando los recursos, con el fin de garantizar el crecimiento económico y el bienestar social de las generaciones presentes y futuras.

El manejo integral de cuencas se puede concebir como la formulación y aplicación en toda la cuenca hidrográfica, tanto aguas abajo como aguas arriba, de un conjunto integrado de acciones en la búsqueda del desarrollo sostenible, minimizando los efectos ambientales negativos sobre el recurso hídrico que la población utiliza aguas abajo. El desarrollo sostenible en manejo de cuencas puede orientar sus acciones hacia:

a) Desarrollo económico, social, cultural que respete las limitaciones del ambiente natural, los valores del hombre y su familia, que sea permanente en el territorio y en el tiempo, en armonía con la naturaleza.

b) Actividad económica diversificada, basada en un desarrollo agropecuario y forestal rentable, que sea menos contaminante y más endógeno en el aprovechamiento de los recursos humanos, naturales y paisajísticos.

c) Urbanización, arquitectura autóctona y paisajismo acorde con el bioclima y que aproveche las tecnologías más adecuadas, en este punto es importante manifestar que la construcción de proyectos residenciales ocasiona trasformaciones ambientales al disminuir la cobertura boscosa, la infiltración del agua en el suelo, modificación de los cauces de agua, y el paisaje natural. Esto está causando la extinción de la variedad y diversidad de fauna y vegetación, poniendo en riesgo las fuentes hídricas que abastecen las ciudades Díaz, T. (2020). Por lo cual también se debe tomar en consideración la planificación de la urbanización.

d) Autogestión a través de la participación, organización, capacitación, educación y divulgación de logros, unido al apoyo decisivo de las instituciones públicas en la construcción de infraestructura y prestación de servicios básicos esenciales

También es importante el reconocer la multifuncionalidad de las cuencas hídricas, y así conciliar los intereses de las diferentes partes y actores implicados en el uso del territorio. De la misma manera se debe recalcar la necesidad de preservar la biodiversidad, los valores naturales y las funciones ecológicas de dichos espacios. El modelo EARP, debe implementarse con un enfoque sostenible, que condescienda la mejora de la calidad de vida de los habitantes locales, resguardando el medio ambiente y no comprometiendo a las generaciones futuras

"Afirman que aunque cada país y cada cuenca requieren soluciones a medida, hay una serie de elementos comunes que estacan nuestra experiencia australiana e internacional y que creemos que son fundamentales para el desarrollo de una gestión sostenible de los recursos hídricos como la importancia de la evidencia científica y el apoyo a la toma de decisiones; importancia de la confianza generada en las partes interesadas y el deseo de resolver los conflictos; compromiso de apoyo y desarrollo a las partes interesadas para enfrentar cambios; reformas institucionales apropiadas, con legislación e incentivos para apoyar el 
cambio de comportamiento; el reconocimiento de que el cambio a largo plazo requiere de tiempo, abriendo nuevas oportunidades para la inversión; entender que el apoyo para el cambio debe ser sostenido con recursos acordes a los retos, incluida la formación de capacidades, consulta y procesos de comunicación." (McDonald, et al. 2016)

Se determina cuáles son los afluentes como quebradas y micro cuencas que alimentan a la demarcación hidrográfica, las comunidades que se desarrollan dentro de la cuenca hidrográfica "Tenguel Alto", La rica, Villa Rica, San Francisco de Muyuyacu, San Vicente de Cachi, Moras, Guena, San Gerardo, La Unión, San Juan de Naranjillas, El Progreso y cuál es su desarrollo económico y cuáles son sus limitaciones para el crecimiento sostenible de las mismas; como es la explotación minera a una cota cercana a las comunidades, provocando daños en las estructuras de las viviendas, la infraestructura vial y en las captaciones de agua para riego y consumo humano. De la información levantada, todas las comunidades tienen los mismos problemas con respecto a la minería y la contaminación de sus cuerpos hídricos por un control defectuoso de las autoridades de control.

\section{b. Análisis socioeconómico}

El estudio aplicado, obtuvo un 53\% de participantes mujeres, y un $47 \%$ de hombres, un $75 \%$ de la población vive en el sector rural, determinándose además que un $80 \%$ dispone de energía eléctrica, un $60 \%$ de agua, un $60 \%$ de servicios higiénicos, y únicamente un $10 \%$ disponen de internet, un $12 \%$ de teléfono, y $15 \%$ de televisión por cable; un $70 \%$ indica que requieren fuerza física para el desempeño de sus actividades laborales,

Al realizar el análisis socioeconómico se determinó que el 70\% de la población poseen un ingreso mensual menor o igual al sueldo básico unificado. El 47,6\% de personas tienen viviendas propias y el $52,40 \%$ dispone de una vivienda arrendada.

El cantón Camilo Ponce Enríquez abastece de medios para el acceso al agua potable, alumbrado público, obras básicas de salud, educación, comunicación, a pesar de ello el agua potable se encuentra accesible solo en el sector urbano. A su vez el cantón no desarrolla industria por lo cual solo es productor de productos primarios, como: cacao, banano entre otros.

La principal actividad económica (36\%) es la minería, seguida de la agricultura (28\%) mientras que el $25 \%$ no posee trabajo fijo.

Camilo Ponce Enríquez se encuentra concesionado el $98 \%$ de su territorio, tanto en concesiones mineras metálicas como no metálicas, sus ríos se encuentran todos concesionados para la explotación de pétreos y de igual manera con libres aprovechamientos para la construcción de la carretera Rio 7- Huaquillas, en la figura 2 se puede observar las concesiones mineras de la zona de la cuenca Tenguel alto, contando con 95 concesiones dentro del polígono planteado de protección principal mente en el sector de San Salvador. 
Figura 2. Concesiones mineras

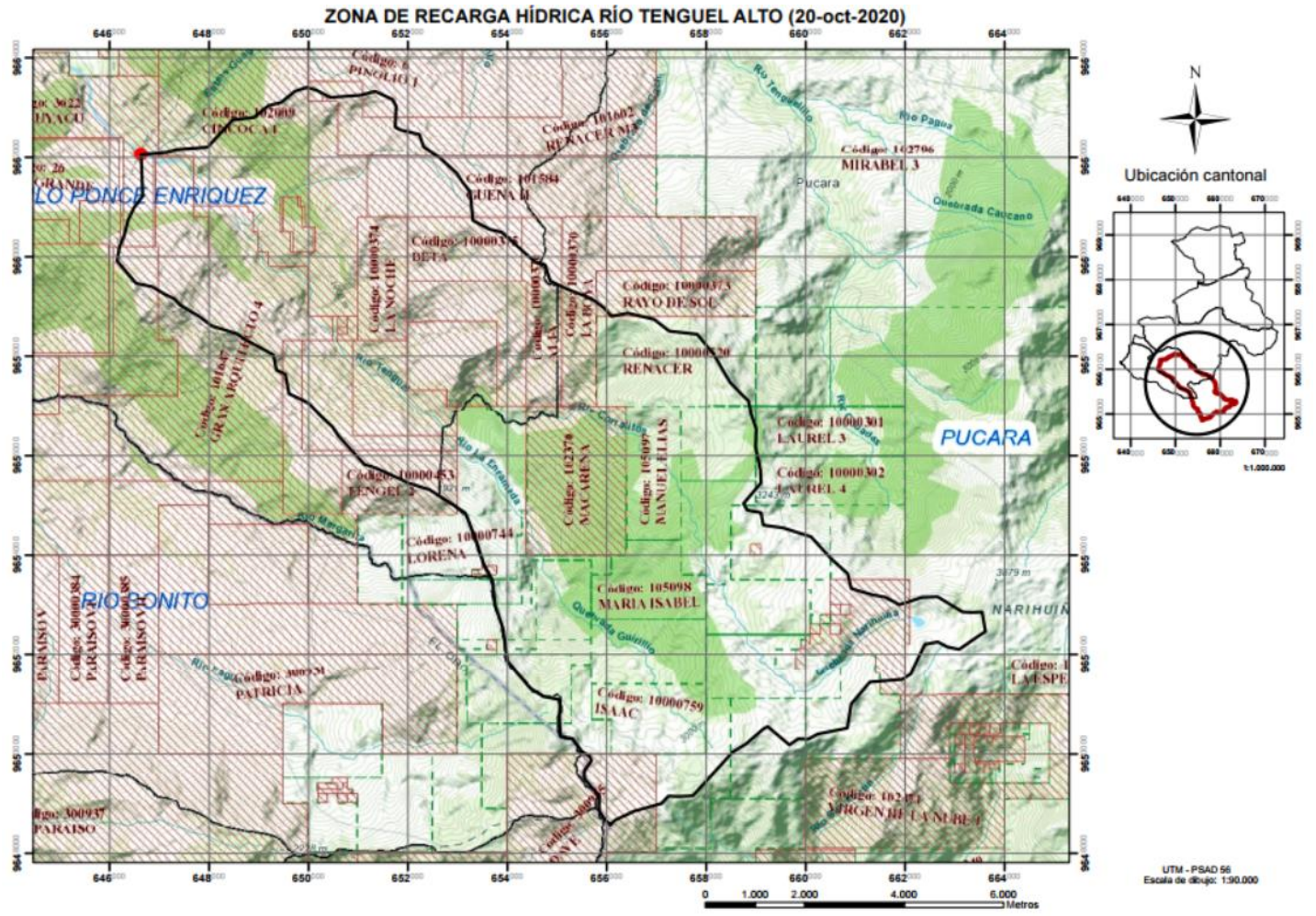

Fuente: El autor

\section{c. Requerimientos de una política integral}

El modelo EARP se enfoca en solucionar los problemas socioeconómicos encontrados en la población aledaña a la cuenca, así como también en cumplir con los lineamientos legislativos del ecuador, así como también a

Dentro de las leyes del manejo de los recursos hidrográficos encontramos dentro de la Constitución de la República del Ecuador en su artículo 262 se establecen las competencias de los gobiernos regionales autónomos el planificar el desarrollo regional y formular los correspondientes planes de ordenamiento territorial, de forma articulada con los planes de mayor jerarquía territorial y el gestionar el ordenamiento de cuencas hidrográficas, así como dar lugar a la creación de consejos de cuenca; en el art. 263 se especifican la competencias exclusivas de los gobiernos provinciales entre las cuales se encuentra: ...1. Planificar el desarrollo provincial y formular los correspondientes planes de ordenamiento territorial, de manera articulada con la planificación nacional, regional, cantonal y parroquial; ...3. Ejecutar, en coordinación con el gobierno regional, obras en cuencas y micro cuencas; 4 . La gestión ambiental provincial; 5. Planificar, construir, operar y mantener sistemas de riego...; y el art. 411 enfocado en el deber del estado de garantizar la conservación, recuperación y 
manejo integral de los recursos hídricos, cuencas hidrográficas y caudales ecológicos asociados al ciclo hidrológico, controlar las actividades que pongan en riesgo la calidad y cantidad de agua, así como el equilibrio de ecosistemas, en especial en las fuentes y zonas de recarga de agua...

El COTAD el art. 411 enfocado en el deber del estado de garantizar la conservación, recuperación y manejo integral de los recursos hídricos, cuencas hidrográficas y caudales ecológicos asociados al ciclo hidrológico, controlar las actividades que pongan en riesgo la calidad y cantidad de agua, así como el equilibrio de ecosistemas, en especial en las fuentes y zonas de recarga de agua. Gestionar el ordenamiento de cuencas hidrográficas a través de la articulación efectiva de los planes de ordenamiento territorial con un enfoque de manejo sustentable e integral de los recursos hídricos.

El Código Orgánico del ambiente el art. 5 garantiza el derecho de la población a vivir en un ambiente sano y ecológicamente equilibrado. el art. 30 los objetivos del Estado respecto a los recursos, se menciona el mantenimiento de los recursos, la regulación e incentivos para la participación de la población, proponer un enfoque integral y sistemático para la gestión de recursos hídricos y la conservación de cuencas hidrográficas en coordinación con la Autoridad Única del Agua.

Ley Orgánica de Recursos Hídricos Usos y Aprovechamiento del Agua el art. 8 se define la gestión integrada de los recursos hídricos y los elementos que se analizan en esta gestión; en el art. 10 se determina el dominio hídrico público del agua en todos sus estados y lugares tanto en los cuales se encuentra este recurso dentro del territorio nacional, es decir, tanto superficial como subterránea; en el art. 27 se establecen los lineamientos para las organizaciones de usuarios de una cuenca.

Respecto a la conservación del agua en el artículo 64 establece los derechos de la naturaleza:

a) La protección de sus fuentes, zonas de captación, regulación, recarga, afloramiento y cauces naturales de agua, en particular, nevados, glaciares, páramos, humedales y manglares;

b) El mantenimiento del caudal ecológico como garantía de preservación de los ecosistemas y la biodiversidad;

c) La preservación de la dinámica natural del ciclo integral del agua o ciclo hidrológico;

d) La protección de las cuencas hidrográficas y los ecosistemas de toda contaminación; $\mathrm{y}$,

e) La restauración y recuperación de los ecosistemas por efecto de los desequilibrios producidos por la contaminación de las aguas y la erosión de los suelos.

Se plantea los problemas legislativos y normativos que se tiene dentro de la cuenca hidrográfica de acuerdo con su ubicación geográfica. El Cantón Camilo Ponce Enríquez 
existe la ordenanza para la protección de fuentes y zonas de recarga hídrica, para las captaciones de agua para consumo humano, pero no se la respeta de acuerdo a los lineamientos del Ministerio de Agua y Ambiente (MAAE) y la Secretaria Nacional del Agua (SENAGUA), ya que otorgan captaciones de agua para uso minero sin el debido control previo y caudal superficial. En el año 2017 en sesión de consejo cantonal cambia el uso de suelo del sector San Salvador de recarga hídrica a industrial minero, con lo que se permite dentro de la cuenca hidrográfica emitir usos de suelo para las distintas fases mineras como la exploración, explotación. Provocando daños en la corteza terrestre y perdida de caudal superficial en la cuenca hídrica del "Tenguel Alto" (Figura 3). En el año 2013 se reforma la ley de minería del ecuador en su artículo 26, excluyendo a los gobiernos autónomos descentralizados en la toma de decisiones sobre las concesiones mineras.

Figura 3. Cambio de Uso de Suelo

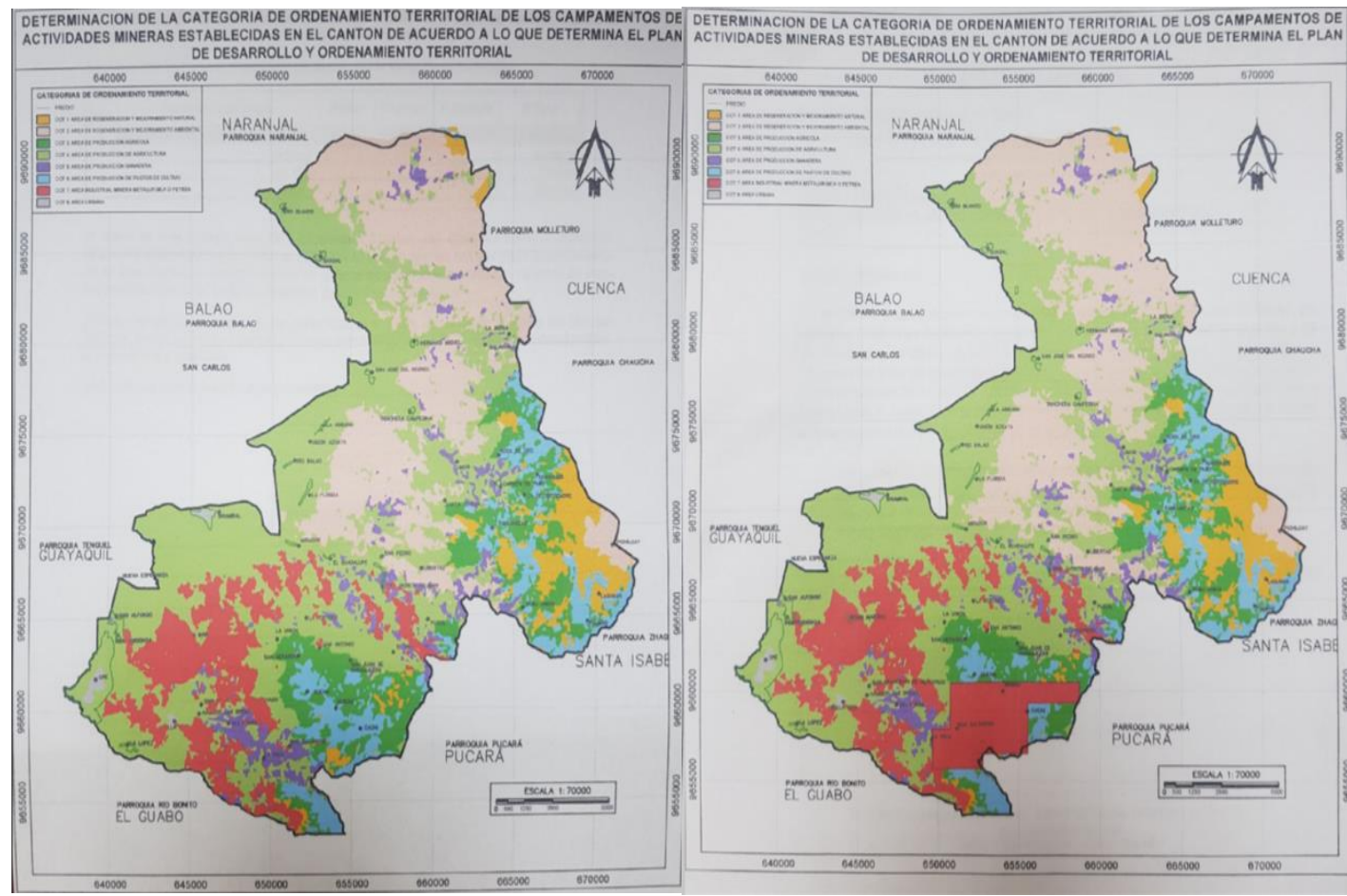

Fuente: Plan de Desarrollo y Ordenamiento Territorial Cantón Camilo Ponce Enríquez

\section{d. Planificación de gestión integral sostenible}

Una vez levantada, tabulada la información el modelo EARP plantea una planificación para la gestión sostenible, en el caso de la cuenca hidrográfica de "Tenguel Alto":

- Gestión del agua generada en la Cuenca Hidrográfica 
En este item se pretende generar una estrategia conjunta entre el ente regulador y la población del cantón Camilo Ponce Enríquez en bien de la conservación del recurso hídrico, así como también de debe prestar especial atención a la remediación ambiental, dando un mayor énfasis en reducción y prevención de la contaminación ambiental.

Principalmente del agua a generada por la fuerte presencia de minería de la zona. La cual puede ser generada mediante una tasa ambiental para el cuidado de la zona en el Pago del Predio Urbano, mediante campañas de conservación por medios digitales dentro del Cantón Camilo Ponce Enríquez, mejorar la infraestructura del sistema de agua potable para la cabecera cantonal, estas alternativas propuestas no han sido aplicadas anteriormente.

- Gestión socio-económico-ambiental de la Cuenca Hidrográfica

Propender la gestión integral del recurso hídrico dando especial importancia al ecosistema, dando prioridad de uso del agua para consumo humano y riego a las comunidades existentes en la cercanía de la cuenca hidrográfica antes que a las sociedades mineras, para así fomentar el la reactivación económica, tomando en cuenta que el sector agrícola es una de las actividades principales en Camilo Ponce Enríquez, por lo antes mencionado se den crear lineamientos que prioricen no potable para el sector agrícola.

La actual administración municipal plantea una alternativa agro minera con la idea de recuperar el sector agrícola y el adecuado control minero contando con un laboratorio municipal para el control de la calidad de agua y el ambiente en las concesiones mineras. De igual manera durante el año 2020 se plantearon polígonos de protección en las comunidades aledañas a la cuenca hidrográfica para que estas no se vean afectadas por la actividad minera.

\section{VALIDACIÓN DEL MODELO EARP}

Para validar el modelo EARP se eligió a 11 profesionales del área, tomando en cuenta los siguientes requisitos: a) Prestigio y profesionalismo reconocido por la sociedad, b) Haber tenido relación laboral docente o practica en desarrollo local o gestión de cuencas, c)5 años como mínimo de experiencia, y, d) formación en el área. Posteriormente se aplica una encuesta en la que los expertos determinan su grado de conocimiento en el tema de la investigación danto todos los expertos un grado de conocimiento mayor a 5 en una escala del 1 al 10.

Como siguiente paso se realizó una evaluación de cada experto sobre su experiencia personal, conocimiento actual de la temática, formación, participación teoría práctica, conocimiento de la literatura especializada y/o publicaciones científicas y la intuición, se aplicó una escala de Alto, Medio y Bajo. Dando como resultado la figura 3. 
Figura 4. Coeficiente de argumentación

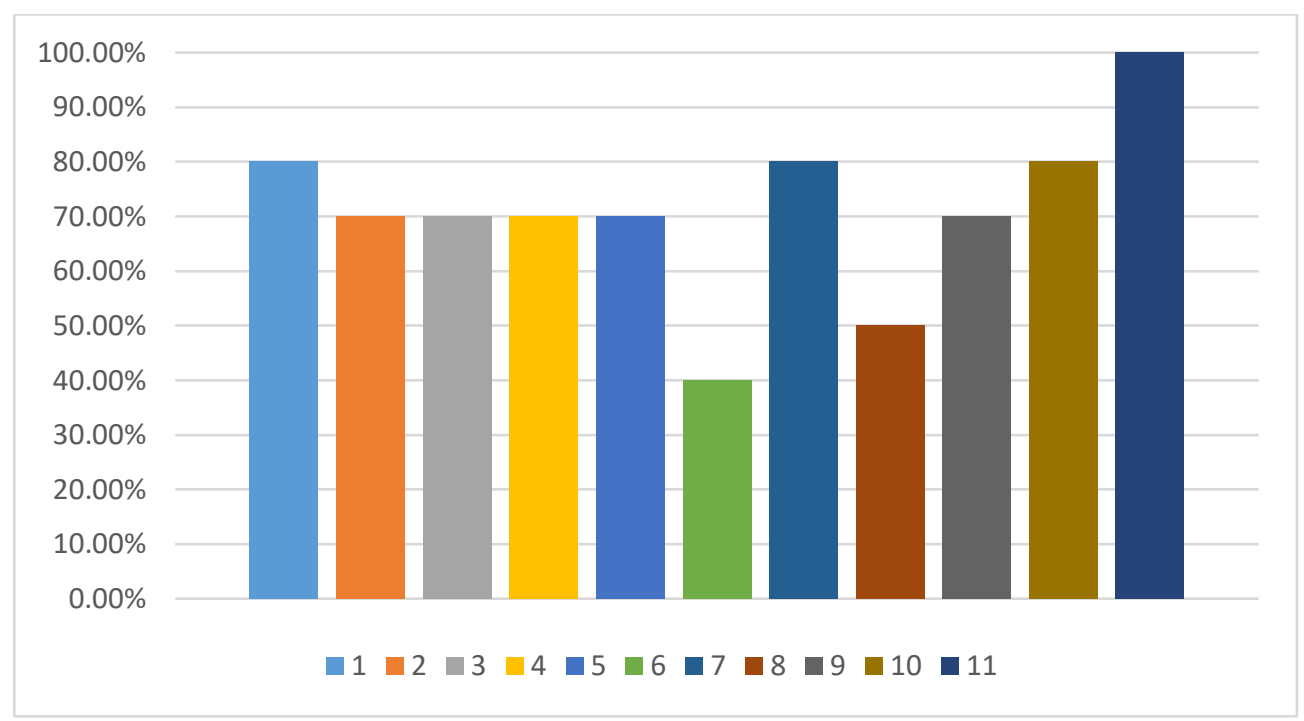

Fuente: El autor

Luego se aplica una media entre el coeficiente de conocimiento y el coeficiente de argumentación para determinar la validez del criterio de los expertos, quedando de esta manera 9 expertos con coeficiente de competencia medio y alto, los cuales procederán a validar el modelo.

Para validar el modelo EARP se aplican 5 niveles de criterios analizan, (1) Totalmente desacuerdo (2) Desacuerdo (3) Neutral (4) De acuerdo (5) Muy de acuerdo y se solicita valorar los siguientes aspectos:

1. Impacto. - Aplicar el Modelo EARP pretende un impacto positivo para mejorar la administración del desarrollo local sostenible en la cuenca hidrográfica de Tenguel Alto.

2. Pertinencia. - El Modelo EARP es apropiado con los objetivos que se persiguen en el mejoramiento la gestión del desarrollo local sostenible en la cuenca hidrográfica de Tenguel Alto

3. Concepción holística-sistémica. - Todas las técnicas que forman parte del Modelo EARP, tienen injerencia directa en los resultados de la gestión del desarrollo local sostenible en la cuenca hidrográfica de Tenguel Alto.

4. Continuidad. - Se encuentra como resultado del análisis de varias metodologías, procedimientos, y modelos existentes en el ámbito internacional y local.

5. Racionalidad. - EARP está basado en el estudios objetivos y críticos de la realidad de la cuenca hidrográfica de Tenguel Alto.

6. Beneficios. - EARP proporciona una gran variedad de beneficios tangibles e intangibles de forma inmediata a las comunidades

7. Innovación. - EARP proporciona un ambiente de intercambio de ideas, al propender en el uso de nuevos métodos y tendencias en el ámbito de las microcuencas, dando como principal objetivo una óptima gestión del desarrollo local sostenible en la cuenca hidrográfica de Tenguel Alto 
8. Integridad. - EARP permite integrar elementos básicos propios de la gestión del desarrollo local sostenible en la cuenca hidrográfica de Tenguel Alto de manera coherente y lógica.

9. Contextualización. - Esta contextualizado acorde a la realidad de las poblaciones aledañas a la cuenca y al desarrollo de la gestión del desarrollo local sostenible en la cuenca hidrográfica de Tenguel Alto

10. Aplicabilidad. - El Modelo EARP es aplicable o puede implementarse en las condiciones actuales de la cuenca hidrográfica de Tenguel Alto.

11. Factibilidad. - Grado de utilización que tiene EARP propuesto dentro de las Instituciones públicas, privadas y la comunidad aledaña a Tenguel Alto.

Al Tabular la encuesta a los expertos, se obtienen los siguientes resultados, fig. 4.

Figura 5. Escala de valoración

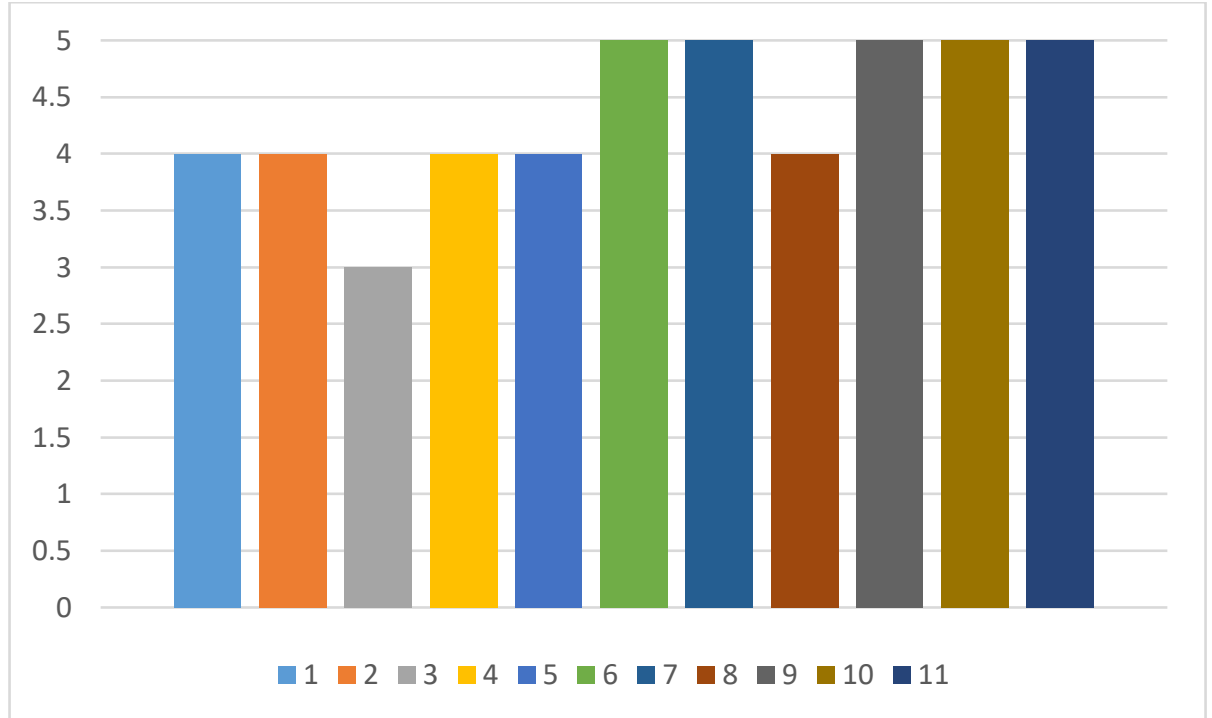

Fuente: El autor

Como se puede observar en la fig. 2, se determina una valoración de 4 sobre 5 para varios aspectos como Impacto, Pertinencia, continuidad, racionalidad e integridad; se determina una valoración máxima de 5 sobre 5 , en los aspectos de Beneficios, Innovación, Contextualización, Aplicabilidad y Factibilidad; finalmente se obtiene un valor de 3 sobre 5 en el aspecto de Concepción holística-sistémica. En general, el modelo EARP propuesto es aceptado por los expertos con una valoración de $87 \%$, lo que valida su aplicabilidad y garantiza su implementación.

\section{Conclusiones.}

- Al realizar una investigación no exhaustiva de las tendencias teóricas sobre la gestión del desarrollo local sostenible con énfasis en el manejo de cuencas hidrográficas, pudimos encontrar las teorías que se representan en la introducción y principalmente los criterios dados por Gaspari et al., (2009) Díaz, T. (2020) y McDonald, et al. (2016), dichos criterios han servido para establecer ciertas pautas de nuestro modelo.

- El modelo EARP es un modelo de gestión sostenible para el manejo de una cuenca hidrográfica, que se basa en 4 puntos estratégicos: Enfoque de la gestión integral 
sostenible de las cuencas hidrográficas, Análisis socioeconómico, Requerimientos de una política integral, Planificación de gestión integral sostenible, así también este modelo propende a la preservación del agua, así como también al desarrollo socioeconómico de la población. El modelo EARP propuesto es aceptado por los expertos con una valoración de $87 \%$, lo que valida su aplicabilidad y garantiza su implementación.

- El modelo EARP tiene la perspectiva centrada en la Gestión del agua generada en la cuenca hidrográfica y Desarrollar una estrategia que involucre a la población en la preservación del recurso hídrico. La gestión socio ambiental Propender la gestión integral del recurso hídrico desde un punto de vista de ecosistema y mejorar la matriz productiva de las comunidades

- El modelo EARP aplicado en la cuenca hidrográfica de "Tenguel Alto" nos indica, las comunidades que se desarrollan alrededor y si estas están progresando de manera sostenible, resguardando el medio ambiente y no comprometiendo a generaciones futuras, de acuerdo al levantamiento socio-económico la principal actividad es la minería, respetando la normativa legal vigente en el país y el Cantón Camilo Ponce Enríquez se plantea polígonos de protección de las comunidades para que estas no se vean afectadas por la actividad minera y de igual manera en las captaciones de agua con la finalidad de no comprometer el recurso hídrico con un control de la calidad de agua y ambiente de la zona.

\section{Referencias Bibliográficas.}

Amendaño Estévez, E. N. (2018). Propuesta de gestión del recurso hídrico de la vertiente La Merced para el desarrollo sostenible, provincia de Pichincha, cantón Mejía, parroquia Cutuglagua (Master's thesis, PUCE-Quito).

Asamblea Nacional del Ecuador. (1 de octubre de 2014). Ley Orgánica de Recurso Hídrico, Usos y Aprovechamiento del Agua. Ley Orgánica de Recurso Hídrico, Usos y Aprovechamiento del Agua. Quito, Pichincha, Ecuador: ANE.

Asamblea Nacional del Ecuador. (01 de octubre de 2017). Código Orgánico del Ambiente. Código Orgánico del Ambiente. Quito, Pichincha, Ecuador: Asamblea Nacional del Ecuador.

Asamblea Nacional del Ecuador. (20 de octubre de 2008). Constitución de la República. Constitución de la República. Quito, Pichincha, Ecuador: Asamblea Nacional.

Barrientos, F. R. (2006). Cuencas hidrográficas, descentralización y desarrollo regional participativo. InterSedes: Revista de las Sedes Regionales, 7(12), 113-125.

Conferencia Internacional sobre el Agua y el Medio Ambiente CIAMA (1992) "Declaración de Dublín e Informe de la Conferencia" Dublin Irlanda Enero 1992.

Congreso nacional de Perú (2009). Ley 29338, Ley de Recursos Hídricos. Lima

Díaz, T. (2020). LA HUELLA HÍDRICA INDICADOR PARA APLICAR LA CIRCULARIDAD DEL AGUA: MODELO DE GESTIÓN SOSTENIBLE PARA PANAMÁ. Cátedra, (17), 66-78. 
ISSN: 2600-5859

Vol. 4, N¹.1, p. 83-100, Febrero, 2021

Jouravlev, A.;(2009) "Acuerdos internacionales para la gestión integrada de cuencas: oportunidades y limitaciones para su implementación" Concepción Chile

Maass, J. (2015). Cuencas de México. https://www.riob.org/sites/default/files/IMG/pdf/cuencas_de_Mexico_web-3.pdf

Martínez Valdés, Y., \& Villalejo García, V. M. (2018). La gestión integrada de los recursos hídricos: una necesidad de estos tiempos. Ingeniería hidráulica y ambiental, 39(1), 58-72.

McDonald, W., Lazarow, N., Wallbrink, P., Keyworth, S., Chiew, F., Jimenez, O., \& Claro, E. (2016) GESTIÓN DE LOS RECURSOS HÍDRICOS EN AUSTRALIA: LECCIONES Y OPORTUNIDADES.

Núñez, M. A. (2011). La cuenca hidrográfica en la gestión integrada de los recursos hídricos. Revista Virtual, 5(1).

Organización de las Naciones Unidas. (2017). Naciones Unidas. Obtenido de ONU, 2015

Peña, Y. A., Pino, M. R. M., \& De León, I. S. (2015). La gestión ambiental de la cuenca del Río Magdalena desde un enfoque socialmente responsable. Amauta, 13(26), 193218.

PNUMA-DHI Centro para el Agua y Medio Ambiente. (2017). Integrated Water Resources Management in Action. Obtenido de Integrated Water Resources Management in Action: http://www.un.org/spanish/waterforlifedecade/iwrm.shtml

Sadoff, C., Greiber, T., Smith, M. \& Bergkamp, G. UICN (2008) "Compartir: Gestionando el agua entre fronteras" Gland Suiza.

Secretaria Nacional del Agua (2010) Acuerdo ministerial 2010-66

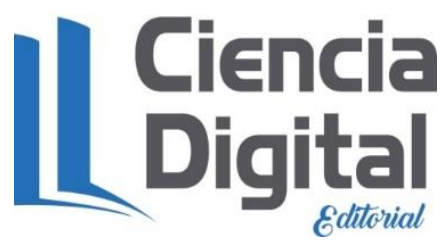




\section{PARA CITAR EL ARTÍCULO INDEXADO.}

Chavez Barriga, S. I., \& Castillo Ortega, Y. (2021). Modelo de gestión sostenible para el manejo de la cuenca hidrográfica "Tenguel Alto" . ConcienciaDigital, 4(1.1), 83-100. https://doi.org/10.33262/concienciadigital.v4i1.1.1548

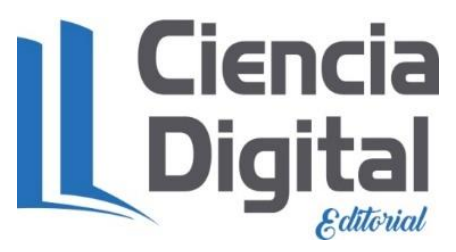

El artículo que se publica es de exclusiva responsabilidad de los autores y no necesariamente reflejan el pensamiento de la Revista Conciencia Digital.

El artículo queda en propiedad de la revista y, por tanto, su publicación parcial y/o total en otro medio tiene que ser autorizado por el director de la Revista Conciencia Digital.

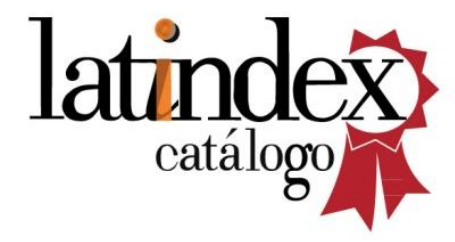

